\title{
Spontaneous Emission in Chaotic Cavities
}

\author{
T Sh Misirpashaev, ${ }^{12} \mathrm{P}$ W Brouwer, ${ }^{1}$ and C W J Beenakker ${ }^{1}$ \\ 'Instituut Lorentz Leiden University, PO Box 95062300 RA Letden The Netherlands \\ ${ }^{2}$ Landau Institute for Theoretıcal Physics, 2 Kosygin Street Moscow 117334, Russia
}

(Recerved 24 June 1997)

\begin{abstract}
The spontaneous emission rate $\Gamma$ of a two-level atom inside a chaotic cavity fluctuates strongly from one point to another because of fluctuations in the local density of modes For a cavity with perfectly conductung walls and an opening containing $N$ wave channels, the distribution of $\Gamma$ is given by $P(\Gamma) \propto \Gamma^{N / 2-1}\left(\Gamma+\Gamma_{0}\right)^{-N-1}$, where $\Gamma_{0}$ is the free-space rate For small $N$ the most probable value of $\Gamma$ is much smaller than the mean value $\Gamma_{0}$ [S0031-9007(97)04001-5]
\end{abstract}

PACS numbers $4250-\mathrm{p}, 0545+\mathrm{b}, 3280-\mathrm{t}$

The modification of the rate of spontaneous emission in a cavity has been a subject of extensive iesearch [18] It was shown that the cavity can both enhance and inhibit the spontaneous emission at microwave and optical frequencies The effect is due to a modification by the environment of the local density of modes at the position of the radiating atom The efforts were concentrated on the fabrication of cavities of prescribed regular shape, the atoms being kept close to nodes or antınodes of the field patterns of the cavity modes

What can be said if the shape of the cavity is not regular and the exact position of the atom is unknown? Irregular cavities have a complicated "chaotic" field pattern, and it becomes difficult to state whether the spontaneous emission rate $\Gamma$ of a panticular atom is increased or decreased with respect to the free-space 1 ate $\Gamma_{0}=d^{2} \omega_{0}^{3} / 3 \pi \epsilon_{0} \hbar c^{3}$ (corresponding to an electric dipole transition with moment $d$, frequency $\omega_{0}$ ) Nevertheless, a precise statement can be made about the statistical distribution of $\Gamma$ The disti ibution is universal, $1 \mathrm{e}$, independent of the shape or size of the cavity, provided it is chaotic

A chaotic cavity is laige compared to the wavelength $\lambda_{0}=2 \pi c / \omega_{0}$, and has a shape such that the light is scattered uniformly in phase space (In a cilcular or cubic cavity, chaotic behavior may still occur because of diffuse boundaly scatteing or due to 1 andomly placed scattering centers ) The only parameter which enters the distribution of $\Gamma / \Gamma_{0}$ is the strength of the coupling of the cavity modes to the outside world We assume that the coupling is via a hole that is small compared to the size of the cavity and transmits a total of $N$ wave channels (For a hole of area $A, N \approx 2 \pi A / \lambda_{0}^{2}$ ) Our result for the distıibution of $\Gamma$ takes the universal form

$$
P(\Gamma) \propto \frac{\Gamma^{N / 2-1}}{\left(\Gamma+\Gamma_{0}\right)^{N+1}},
$$

shown in Fig 1 for several values of $N$ The disti1bution eventually becomes natrow and Gaussian for $N \gg 1$, while it is still bioad and stiongly non-Gaussian for $N$ as laige as 10 The mean value of $\Gamma$ equals $\Gamma_{0}$, but the most probable value is smaller than $\Gamma_{0}$
As a possible experimental setup, one can imagine an array of cavities, each containing a few excited atoms, or a single cavity containıng many excited atoms (widely separated so that they decay independently) The array of cavities might occur naturally in a porous material Let $n(t)$ be the number of atoms that has not decayed by the time $t$ The fraction $n(t) / n(0)$ is the Laplace transform $\int_{0}^{\infty} d \Gamma P(\Gamma) \exp (-\Gamma t)$ of the distribution (1), which is a confluent hypergeometric function A time-resolved measuiement of the emitted intensity yields $n(t)$ and theieby the probability distribution $P(\Gamma)$ Fluctuations of the spontaneous emission rate give rise to an algebraic decay $n(t) \propto t^{-N / 2}$ for large $t$, instead of the usual exponential decay $\propto \exp \left(-\Gamma_{0} t\right)$

We proceed with the derivation of $\mathrm{Eq}$ (1) We assume that the system is in the perturbative regime [9], so that the rate of spontaneous emission is given by the Fermi

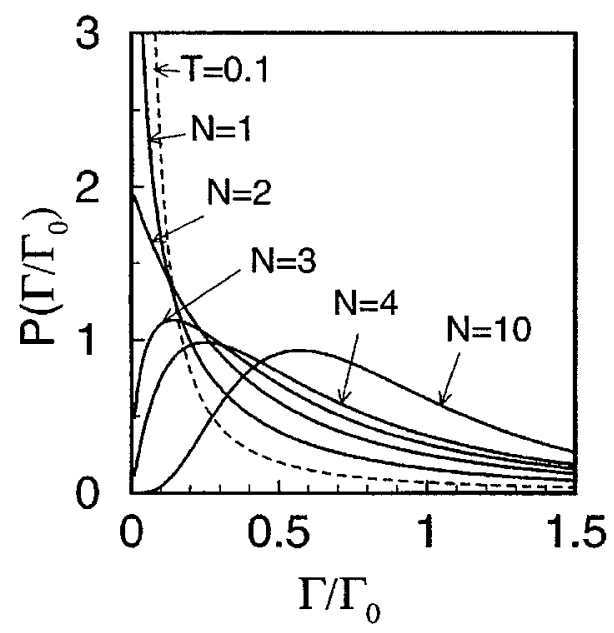

FIG 1 Probability distribution of the spontaneous emission late $\Gamma$ (normalized by the free-space rate $\Gamma_{0}$ ), as given by Eq (1) for several values of the number $N$ of wave channels transmitted by the hole in the cavity For $N \geqslant 2$ the distribution reaches its maximum at a rate $\Gamma=\Gamma_{0}(N-$ $2) /(N+4)$ that is smaller than the mean value $\Gamma_{0}$ The valiance of $\Gamma$ diverges for $N \leq 2$ and equals $4 \Gamma_{0}^{2} /(N-2)$ for larger $N$ The dashed curve is the result (15) for a hole much smaller than a wavelength (transmittance $T=01$ ) 
We first compute the distribution of the vector $\vec{v}=$ $B^{-1} \vec{u}$, which is given by Eq (13) with the delta function replaced by $\delta\left(\vec{v}-B^{-1} \vec{u}\right)$ The result is $P(\vec{v}) \propto$ $\left(1+|\vec{v}|^{2}\right)^{-N-1} \quad$ Because of rotational invaliance of the Gaussian distribution for $\vec{u}$, the distıbutions of $x$ and $|\vec{v}|^{2}$ are the same Hence $P(x)=\int d \vec{v} P(\vec{v}) \delta\left(x-|\vec{v}|^{2}\right) \propto$ $x^{N / 2-1}(1+x)^{-N-1}$ This is the result (1) announced in the introduction and plotted in Fig 1 It decieases monotonically for $N \leq 2$, and has a maximum at nonzeio $\Gamma$ for larger $N$

Th1s calculation holds for the so-called orthogonal symmetry class (symmetry index $\beta=1$ ), relevant for optical systems with time-ieversal symmetry The local density of states for systems with broken time-1eversal symmetry (unitary class, $\beta=2$ ) or with broken spintotational symmetry (symplectic class, $\beta=4$ ) is ielevant in condensed matter physics We have repeated our calculations for $\beta=2,4$ and found $P_{N}^{(\beta)}=P_{\beta N}^{(1)}(\lambda)$, with $P^{(1)}(x)$ given by $\mathrm{Eq}(1)$

So far we have assumed that the hole in the cavity fully transmits at least one wave channel, so that the transmittance $T$ of the hole (the ratio of the transmitted and incident power) is $\geq 1$ If the hole is smallet than a wavelength, then $T$ becomes $<1$ The scattering matrix $S(T)$ of the cavity coupled by a hole with tiansmittance $T<1$ can be expressed in terms of the scattering matrix $\left.S\right|_{T=1}$

$$
S(T)=\frac{\left.S\right|_{T=1}+\sqrt{1-T}}{1+\left.S\right|_{T=1} \sqrt{1-T}}
$$

To find the distribution of the local density of modes, we start from Eq (8) with $S$ replaced by $S(T)$, repeat similar steps, and average over $\left.S\right|_{T=1}=e^{l \phi}$ at the end The result is

$$
\begin{aligned}
P(x)= & \frac{2}{\pi^{2} \sqrt{x T}} \int_{0}^{\pi} d \phi \\
& \times \frac{\sqrt{2-T+2 \sqrt{1-T} \cos \phi}}{[1+x(2-T+2 \sqrt{1-T} \cos \phi) / T]^{2}},
\end{aligned}
$$

plotted also in Fig 1 (dashed line, for $T=0$ 1) It decreases monotonically for any $T<1$

The variance $\left\langle\left(\Gamma-\Gamma_{0}\right)^{2}\right\rangle$ diverges if $N \leq 2$ but the diveigency is removed when we take into account the condition of applicability of the Ferm golden rule (2) The peiturbative treatment is valid as long as the decay rate $\Gamma$ of the excited atom remains smaller than the width $\gamma_{\mu}$ of the cavity modes contributing to the decay Estimating the width of the main contributing mode as $1 / \rho \mathcal{V}=\Gamma_{0} / \Gamma \rho_{0} V$, we get a condition $\Gamma \ll\left(\Gamma_{0} / \rho_{0} V\right)^{1 / 2} \quad$ Therefore, any divergent contribution of the large $\Gamma$ tail should be cut off at $\Gamma \simeq\left(\Gamma_{0} / \rho_{0} V\right)^{1 / 2}$ The werght of the tail is negligibly small provided $\left(\Gamma_{0} / \rho_{0} V\right)^{1 / 2} \gg \Gamma_{0}$, hence if $\Gamma_{0} \rho_{0} V=d^{2} \omega_{0}^{5} V / 9 \pi^{3} \epsilon_{0} \hbar c^{6} \ll 1 \quad$ To estımate this parameter, we write $d=z e a_{B} \quad\left(a_{B}\right.$ is the Bohr radius), $\omega_{0}=2 \pi c / \lambda_{0}, \quad V=L^{3} \quad$ Then $\Gamma_{0} \rho_{0} V \approx 321 z^{2} a_{B}^{2} L^{3} / \lambda_{0}^{5}$ is close to 1 for $z=017$, $L=053 \mathrm{~mm}, \lambda_{0}=530 \mathrm{~nm}$ We can get large $100 \mathrm{~m}$ for applicability of Eqs (1) and (15) by going to weaker (possibly magnetic) dipoles, smaller cavities, or larger (possibly miciowave) wavelengths

We conclude with a comparison with previous work on the local density of states in chaotic cavities [1113] That work was motivated by different physical applications (Knight shift in NMR or optical absorption) Our application is in a sense dual to that of Ref [13], where complicated electronic states interact with simple 1adiation states Instead, we have the simplest possible electronic system - a two level atom - and a complicated structure of radiation modes In Refs [11-13] it was assumed that the cavity was coupled to the outside via a tunnel barrier of laige area In this case statistical fluctuations in the broadening of the levels $\gamma_{\mu}$ (from level to level and from cavity to cavity) can be ignored In the case of a relatively small opening, considered here, fluctuations of the $\gamma_{\mu}$ 's are essential The resulting distribution (1) of the local density of modes tuins out to be very simple, compared with the result of Ref [13] (involving a fivefold integial in the case of unbroken tıme-reveisal symmetry) We obtained oul result within the framework of 1 andom-matrix theory It would be interesting to see if it can be repioduced using the supersymmetıy technique of Refs [11,13]

This work was supported by the Nederlandse Or ganısatıe voor Wetenschappelıjk Onderzoek (NWO) and the Stichting voor Fundamenteel Onderzoek der Materie (FOM)

[1] D Kleppner, Phys Rev Lett 47, 233 (1981), R G Hulet, E S Hilfer, and D Kleppner, Phys Rev Lett 55, 2137 (1985)

[2] P Goy, J M Raimond, M Gioss, and S Haroche, Phys Rev Lett 50, 1903 (1983)

[3] G Gabrielse and H Dehmelt, Phys Rev Lett 55, 67 (1985)

[4] W Jhe, A Andeison, E A Hinds, D Meschede, L Mor, and $S$ Haloche, Phys Rev Lett 58, 666 (1987)

[5] D J Heinzen, J L Childs, J E Thomas, and M S Feld, Phys Rev Lett 58, 1320 (1987)

[6] F De Ma1tin1, G Innocent,, G R Jacobovitz, and P Matalon, Phys Rev Lett 59, 2955 (1987)

[7] S M Barnett and R Loudon, Phys Rev Lett 77, 2444 (1996)

[8] S D Biorson and P M W Skovgaard, in Optical Pro cesses in Microcavities edited by R K Chang and A J Campillo (Wol ld Scientific, Singapore, 1996), A J Campillo, J D Eversole, and H -B Lin, ibid

[9] S Haroche, in Fundamental Systems in Quantum Opttcs edited by J Dalıbaid, J M Raimond, and J Zinn-Justın (North Holland, Amsterdam, 1992) 
[10] R. Sprik, B. A. van Tiggelen, and A. Lagendy]k, Europhys. Lett. 35, 265 (1996); A. Lagendijk and B. A. van Tiggelen, Phys. Rep. 270, 143 (1996).

[11] K. B. Efetov and V. N. Prigodın, Phys. Rev. Lett. 70, 1315 (1993).

[12] C. W. J. Beenakker, Phys. Rev. B 50, 15170 (1994).

[13] N. Tanıguchı and V. N. Prigodin, Phys. Rev. B 54, 14305 (1996).

[14] J. J.M. Verbaarschot, H.A. Werdenmuller, and M.R. Zirnbauer, Phys. Rep. 129, 367 (1985).
[15] M. Buttıker, A. Prêtre, and H. Thomas, Phys. Rev. Lett. 70, 4114 (1993).

[16] E.P. Wigner, Phys. Rev. 98, 145 (1955); F. T. Smith, Phys. Rev. 118, 349 (1960).

[17] Y. V. Fyodorov and H.-J. Sommers, Phys. Rev. Lett. 76, 4709 (1996); J. Math. Phys. (N.Y.) 38, 1918 (1997).

[18] P. W. Brouwer, K. M. Frahm, and C.W.J. Beenakker, Phys. Rev. Lett. 78, 4737 (1997). 